\title{
Very High Frequency Silicon Nanowire Electromechanical Resonators
}

\author{
X. L. Feng, ${ }^{\dagger}$ Rongrui He, ${ }^{\ddagger}$ Peidong Yang, ${ }^{\ddagger}$ and M. L. Roukes ${ }^{\star}, \dagger$ \\ Kavli Nanoscience Institute, California Institute of Technology, Mail Code 114-36, \\ Pasadena, California 91125, Department of Chemistry, University of California, \\ Materials Science Division, Lawrence Berkeley National Laboratory, Berkeley, \\ California 94720
}

Received March 22, 2007

\begin{abstract}
We demonstrate very high frequency (VHF) nanomechanical resonators based upon single-crystal silicon nanowires (SiNWs), which are prepared by the bottom-up chemical synthesis. Metallized SiNW resonators operating near $200 \mathrm{MHz}$ are realized with quality factor $Q \approx 2000-2500$. Pristine SiNWs, with fundamental resonances as high as $215 \mathrm{MHz}$, are measured using a VHF readout technique that is optimized for these high resistance devices. The pristine resonators provide the highest $Q$ 's, as high as $Q \approx 13100$ for an $80 \mathrm{MHz}$ device. SiNWs excel at mass sensing; characterization of their mass responsivity and frequency stability demonstrates sensitivities approaching 10 zeptograms. These SiNW resonators offer significant potential for applications in resonant sensing, quantum electromechanical systems, and high frequency signal processing.
\end{abstract}

Nanoelectromechanical systems (NEMS), particularly nanomechanical resonators vibrating at high frequencies, ${ }^{1}$ are being actively explored for applications including resonant sensors for ultrahigh-resolution mass sensing, ${ }^{2}$ force detection, ${ }^{3}$ quantum electromechanics, ${ }^{4}$ electromechanical signal generation and processing, ${ }^{5}$ and high-speed logic and computation. ${ }^{6}$ These NEMS resonators are usually made by topdown lithographic techniques and surface nanomachining, which together enable realization of nanomechanical devices with considerable complexity and functionality. By contrast, chemical-synthesis-based bottom-up approaches now provide nanowires with high crystalline quality, perfectly terminated surfaces, and sizes down to the molecular scale. These represent a new class of building blocks for NEMS resonators that offer unique attributes. Si nanowires (SiNWs) are, perhaps, among the most intriguing given silicon's preeminent role in micro- and nanoelectronics and as a structural material for micro- and nanoelectromechanical systems. ${ }^{7}$ Development of SiNW-NEMS, however, has been impeded by difficulties in suspending SiNWs to give them mechanical freedom and in subsequent device integration. Recently, a hybrid process has been developed to fabricate SiNWs suspended over microtrenches by employing vapor-liquidsolid (VLS) epitaxial growth. ${ }^{8}$ This device geometry facilitates the direct probing of mechanical properties of SiNWs via static deflection. ${ }^{9,10}$ In this Letter, we describe the first

* Corresponding author. E-mail: roukes@caltech.edu. Telephone: 1-626395-2916.

California Institute of Technology.

$\doteqdot$ University of California, Berkeley. demonstration of resonant mechanical devices operating at very high frequencies (VHF) that are based on such suspended SiNWs. We demonstrate robust SiNW resonators vibrating at frequencies greater than $200 \mathrm{MHz}$. Furthermore, comprehensive measurements of the resonance characteristics, quality factors, and resonator frequency stability show that these bottom-up SiNWs provide excellent performance. These devices expand and advance prospects for NEMS resonator technologies and enable new possibilities for applications.

Parts $\mathrm{a}$ and $\mathrm{b}$ of Figure 1 show the typical suspended SiNWs in microtrenches. The detailed synthetic procedure via VLS epitaxial growth has been described in ref 8. Briefly, the SiNW begins crystal nucleation on one trench wall, axially grows along the $\langle 111\rangle$ direction, self-welds at the opposite trench wall, and then usually continues to grow along a backward $\langle 111\rangle$ direction. In the present work, the microtrenches are $\sim 4 \mu \mathrm{m}$ deep and the trench widths are varied from 1.6 to $3.0 \mu \mathrm{m}$. It needs to be emphasized that, different from top-down fabricated nanobeams, chemically synthesized SiNWs have well-faceted side surfaces. Figure $1 \mathrm{c}$ demonstrates the hexagonal cross section of these singlecrystal SiNWs, which are enclosed by the $\{112\}$ crystalline planes. The geometric and crystalline orientation of the hexagonal cross section with respect to the (110) substrate is illustrated in the left inset of Figure 1a. The lateral dimension $d$ measured in the SEM is taken as the SiNW width. Note that a hexagonal cross section has a flexural moment of inertia given by $I_{11}=I_{22}=I=5 \sqrt{3} d^{4} / 144$, 


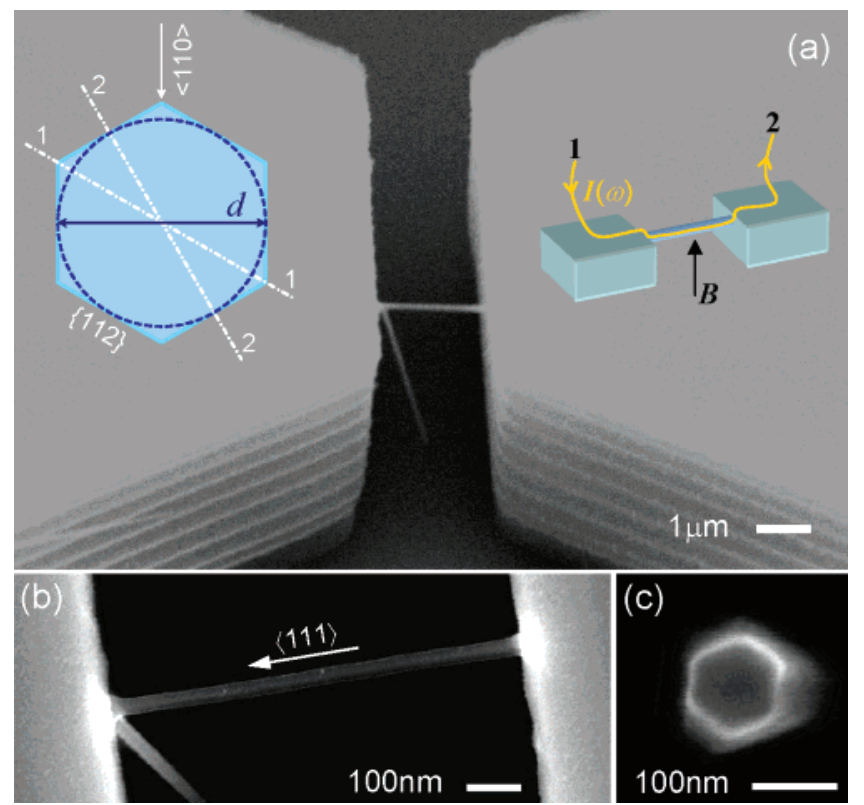

Figure 1. Scanning electron micrographs of pristine suspended SiNWs. (a) A typical silicon nanowire (SiNW) grown in a microtrench defined by two heavily doped supporting pads. Insets in (a): (left) orientation of the SiNW cross section; (right) illustration of magnetomotive transduction of a SiNW. (b) A closeup top view of a straight SiNW with a constant width of about 60 $\mathrm{nm}$. The white arrow indicates the growth front of the nanowire. (c) The hexagonal cross section of a SiNW, enclosed by wellfaceted $\{112\}$ planes.

which, in the absence of tension, leads to a fundamental flexural mode resonance frequency of

$$
f_{0} \equiv \frac{\omega_{0}}{2 \pi}=0.9395 \frac{d}{L^{2}} \sqrt{\frac{E_{\mathrm{Y}}}{\rho}}
$$

where $L, E_{\mathrm{Y}}$, and $\rho$ are the length, Young's modulus, and density of the SiNW, respectively.

The Si pads are heavily doped with boron, yielding a resistivity of $\sim 0.01 \Omega \cdot \mathrm{cm}$. The resistivity of these pristine SiNWs ranges from $\sim 0.01$ to $\sim 0.001 \Omega \cdot \mathrm{cm}$ depending upon the doping and growth process details. Measurements show that the electrical junctions between the SiNWs and the trench sidewalls are Ohmic. Electrical contacts (wire bonds) are made from selected devices to a radio frequency (RF) circuit board, and the sample is then loaded into an $\mathrm{RF} /$ microwave-compatible chamber that also functions as a low-temperature cryostat.

A central challenge for engineering SiNW NEMS resonators is obtaining efficient excitation and transduction of the high frequency mechanical response. Existing techniques widely used in MEMS (microelectromechanical systems) are being pursued with other NEMS resonators; among these are electrostatic ${ }^{11,12}$ and piezoelectric ${ }^{13}$ schemes for excitation and optical ${ }^{11}$ and capacitive ${ }^{12}$ schemes for detection. The efficiency of these schemes generally decreases substantially as we scale devices from the size regime of MEMS to that of NEMS. For example, in capacitive detection of nanobeam and nanowire NEMS resonators, the capacitive coupling to the device becomes miniscule $\left(<10^{-18} \mathrm{~F}\right)$ for dimensions of $100 \mathrm{~nm}$ and below. For this reason, it is not straightforward to apply this transduction scheme for NEMS devices operating from the mid-VHF range upward. At lower frequencies, $\sim 10-20 \mathrm{MHz}$, capacitive transduction has been successfully demonstrated with NEMS beams, albeit with considerable engineering effort. ${ }^{12}$ On the other hand, novel transduction techniques, ones not previously employed for MEMS, are being developed for NEMS resonators. Among these are magnetomotive transduction, ${ }^{14-16}$ single-electron transistor detectors, $, 13,16,17$ sensing via a quantum or atomic point contact, ${ }^{18,19}$ and various combinations of the above. ${ }^{16,18,19}$ These are motivated by the need for increased bandwidth and the desire to pursue ultrasensitive, ultimately quantumlimited detection. This drives the development of highly miniaturized devices with critical performance specifications; the ultimate performance is attained with operation at $\mathrm{mK}$ temperatures.

We find that magnetomotive transduction is well suited to the SiNW-in-microtrench configuration displayed in Figure 1. The device design enables VHF and higher frequency operation and appears scalable into the ultrahigh frequency range (UHF, $300 \mathrm{MHz}-1 \mathrm{GHz}) .{ }^{15}$ To measure the devices, we pass a RF current $\boldsymbol{I}(\omega)$ through the SiNW (of length $L$ ), which, in the presence of an applied magnetic field $B$, generates a Lorentz force $\boldsymbol{F}(\omega)=L B \boldsymbol{I}(\omega)$ (Figure 1a, right inset). This force excites vibration of the SiNW, which in turn induces an electromotive force (EMF) $V_{\mathrm{EMF}}(\omega)$ due to the wire's transverse motion in the $B$ field. The SiNW NEMS resonance, transduced as an EMF developed along the SiNW, is then characterized by measuring the transmitted power via RF network analysis. The flexural motion in the $B$ field induces a frequency-dependent EMF given as

$$
\boldsymbol{V}_{\mathrm{EMF}}(\omega)=B L \boldsymbol{v}(\omega) \cdot \frac{1}{L} \int_{-L / 2}^{L / 2} u_{0}(x) \mathrm{d} x=j \omega \eta B L \boldsymbol{a}(\omega)
$$

Here $\boldsymbol{v}(\omega)$ and $\boldsymbol{a}(\omega)$ are, respectively, the velocity and displacement at the SiNW's midpoint $(x=0)$. The midpoint displacement can be described by a damped harmonic oscillator model

$$
\boldsymbol{a}(\omega)=\frac{B L \boldsymbol{I}(\omega)}{M_{\mathrm{eff}}\left[\left(\omega_{0}^{2}-\omega^{2}\right)+j \omega_{0} \omega / Q\right]}
$$

provided that we interpret $M_{\text {eff }}$ as a mode-specific effective mass and $Q$ is the modal quality factor. Here $u_{0}(x)$ is the normalized mode shape (with the midpoint amplitude normalized to unity, i.e., $\left.u_{0}(x)=1\right), u_{0}(x)=0.8827$ cos$(4.73 x / L)+0.1173 \cosh (4.73 x / L)$ for $x \in[-L / 2, L / 2]$, and thus the modal weighting factor is $\eta=1 / L \int_{-L / 2}^{L / 2} u_{0}(x) \mathrm{d} x=$ 0.5232 in eq 2 . Note that $\boldsymbol{I}(\omega), \boldsymbol{F}(\omega), \boldsymbol{V}_{\mathrm{EMF}}(\omega), \boldsymbol{v}(\omega)$, and $\boldsymbol{a}(\omega)$ are all complex variables in this formulation.

We first demonstrate VHF resonators based on metallized SiNWs. Because the pristine SiNWs we have investigated typically have two-terminal resistances in the range of $1-100$ $\mathrm{k} \Omega$, they present a severe impedance mismatch to standard 

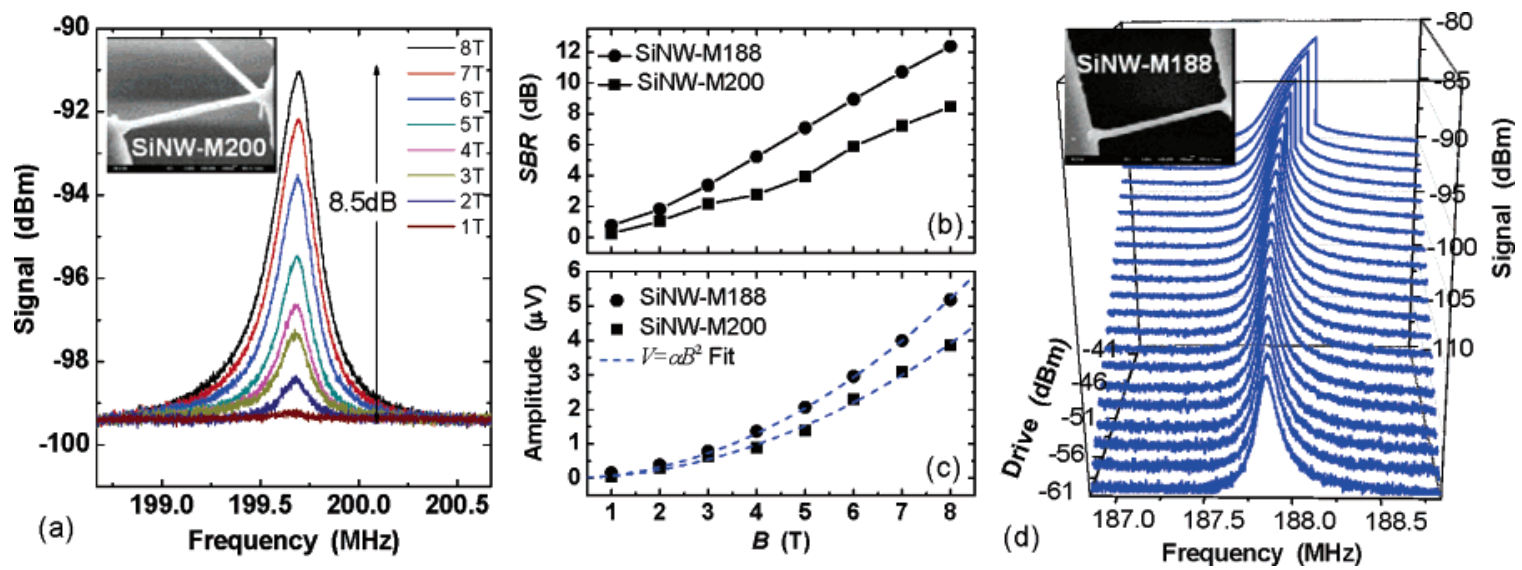

Figure 2. Measured resonance characteristics of the metallized SiNW very high frequency (VHF) resonators. (a) Magnetomotively transduced response (referred-to-input, RTI, of the first-stage amplifier) from the SiNW-M200 device at varying $B$ field (RF drive to device is -46 $\mathrm{dBm}$ ). (b) Achieved detection efficiency, signal-to-background ratio (SBR) in dB, in the measurement and (c) the actual voltage signal amplitude of the SiNW resonances displaying the $B^{2}$ dependence expected from magnetomotive transduction. (d) Magnetomotive response (RTI) from the SiNW-M188 device as RF drive to the device sweeps from -61 to $-41 \mathrm{dBm}$ (at $B=6 \mathrm{~T}$ ).

$50 \Omega$ RF components. This significantly compromises the efficiency of actuation and transduction, especially at VHF and beyond. To overcome this issue, we first metallize SiNWs to obtain a transducer impedance close to $50 \Omega$. This involves deposition of $30 \mathrm{~nm} \mathrm{Al}$ and $5 \mathrm{~nm}$ Ti layers on the SiNWs. The typical resistances obtained for metallized SiNWs are $\sim 60-80 \Omega$ at room temperature and are hence well-matched to $50 \Omega$ at low temperatures. The metallized SiNWs are then measured in vacuum at $T \approx 25 \mathrm{~K}$ using magnetomotive transduction by means of a carefully optimized readout technique. ${ }^{5}$ This is important because, in practical detection schemes, especially those at high frequencies, the NEMS resonance signal is invariably embedded in a strong background response. This response originates from, for example, reflected and standing waves induced by impedance mismatch, parasitic impedances, and other nonidealities. To quantify detection efficiency, we define the signal-to-background ratio (SBR) as the ratio between onresonance and off-resonance signal power, SBR $=10 \mathrm{log}$ $\left(P_{\text {on-res }} / P_{\text {off-res }}\right)$ in $\mathrm{dB}$. This quantity can be directly measured via RF network analysis. As a simplified example, a $10 \mathrm{pW}$ $\left(1 \mathrm{pW}=10^{-12} \mathrm{~W}\right)$ signal on a $1 \mathrm{nW}$ background level yields $\mathrm{SBR}=10 \log (1.01 \mathrm{nW} / 1 \mathrm{nW}) \approx 0.04 \mathrm{~dB}$, which in practical measurements is typically unresolvable, while on a $1 \mathrm{pW}$ background, the same $10 \mathrm{pW}$ signal will emerge as a readily observable peak with $\mathrm{SBR}=10 \log (11 / 1) \approx 10.4 \mathrm{~dB}$. This illustrates the importance of minimizing the off-resonance background response. Our recently developed backgroundnulling techniques and readout schemes for UHF NEMS ${ }^{5}$ are employed in the present work to read out the signals from SiNW resonators that are intrinsically at very low levels.

Figure 2 demonstrates the measured characteristics from two metallized SiNW resonators, SiNW-M200, with dimensions $L=2.25 \mu \mathrm{m}, d=142 \mathrm{~nm}$ and fundamental resonance at $f_{0} \approx 200 \mathrm{MHz}$, and SiNW-M188, with $L=2.1 \mu \mathrm{m}, d=$ $118 \mathrm{~nm}$ and $f_{0} \approx 188 \mathrm{MHz}$. With careful calibration, our measurements yield accurate values for both the signal and the background levels; this enables evaluation of the signals referred to the input (RTI) of the first-stage amplifier, i.e., at the juncture between the nanowire electrodes and the readout amplifier. Figure 2a shows data from the SiNWM200 resonator, RTI, as the magnetic field is varied from 1 to $8 \mathrm{~T}$, while the RF drive is fixed at $P_{\mathrm{drv}}=-46 \mathrm{dBm}$. Note the excellent $\mathrm{SBR} \approx 8.5 \mathrm{~dB}$ obtained at $B=8 \mathrm{~T}$, which is a direct consequence of our carefully optimized readout. This yields a nearly frequency-independent background of -99.5 $\mathrm{dBm}(0.11 \mathrm{pW})$, which is obtained without any softwarebased normalization. With a resonance peak of $-91 \mathrm{dBm}$ $(0.79 \mathrm{pW})$ at $B=8 \mathrm{~T}$, the SiNW-M200 resonator yields a signal of absolute power $0.68 \mathrm{pW}$. Similar data is obtained for SiNW-M188 by varying the applied $B$ field at a fixed RF drive to device of $P_{\mathrm{drv}}=-54 \mathrm{dBm}$. In the data from SiNW-M188 (not presented here for brevity), we find a maximum $\mathrm{SBR}=12.5 \mathrm{~dB}$ is achieved at $8 \mathrm{~T}$, with a background level of only $-102.8 \mathrm{dBm}(0.05 \mathrm{pW})$. The resonance peak at $188 \mathrm{MHz}$ rises above this background, with its peak at $-90.3 \mathrm{dBm}(0.93 \mathrm{pW})$, corresponding to an absolute signal of $0.88 \mathrm{pW}$. Measured SBR and root-meansquare (rms) voltage signal amplitude $V_{\text {rms }}\left(\omega_{0}\right)$ of the resonance at varied $B$ field are shown in parts $\mathrm{b}$ and $\mathrm{c}$ of Figure 2, respectively. The $\sim 10 \mathrm{~dB}$ SBR we have attained for $\sim 200 \mathrm{MHz}$ metallized SiNWs is striking in comparison with previously reported results: $\sim 1 \mathrm{~dB}$ SBRs for $\sim 10 \mathrm{MHz}$ NEMS beams, ${ }^{12} \sim 2 \mathrm{~dB}$ SBRs for $\sim 200 \mathrm{MHz}$ beams, ${ }^{20}$ and $\sim 0.5$ to $\sim 0.005 \mathrm{~dB}$ SBRs typically observed for UHF beams at $\geq 300 \mathrm{MHz}$ and $1 \mathrm{GHz} \cdot{ }^{15,21} \mathrm{In}$ all of these aforementioned cases, the beams were metallized, and significant efforts were made to optimize the SBR.

Following eqs 2 and 3, we find a good fit of the data in Figure $2 \mathrm{c}$ to the quadratic form $V=\alpha B^{2}$ where $\alpha=(0.5232) L^{2} I Q /\left(M_{\mathrm{eff}} \omega_{0}\right)$. From the fit, we determine the rms RF current passing through the devices on resonance is $I_{\text {rms }}\left(\omega_{0}\right)=0.75 \mu \mathrm{A}$ for SiNW-M188 with a measured $Q \approx$ 2500 , and $I_{\mathrm{rms}}\left(\omega_{0}\right)=1.02 \mu \mathrm{A}$ for SiNW-M200 with $Q \approx$ 2000. As shown in Figure 2d with data from SiNW-M188, by increasing $P_{\mathrm{drv}}$ from -61 to $-41 \mathrm{dBm}$ at fixed $B=6 \mathrm{~T}$, we observe that the resonance response transitions from a linear to a strongly nonlinear regime. The nonlinear fre- 

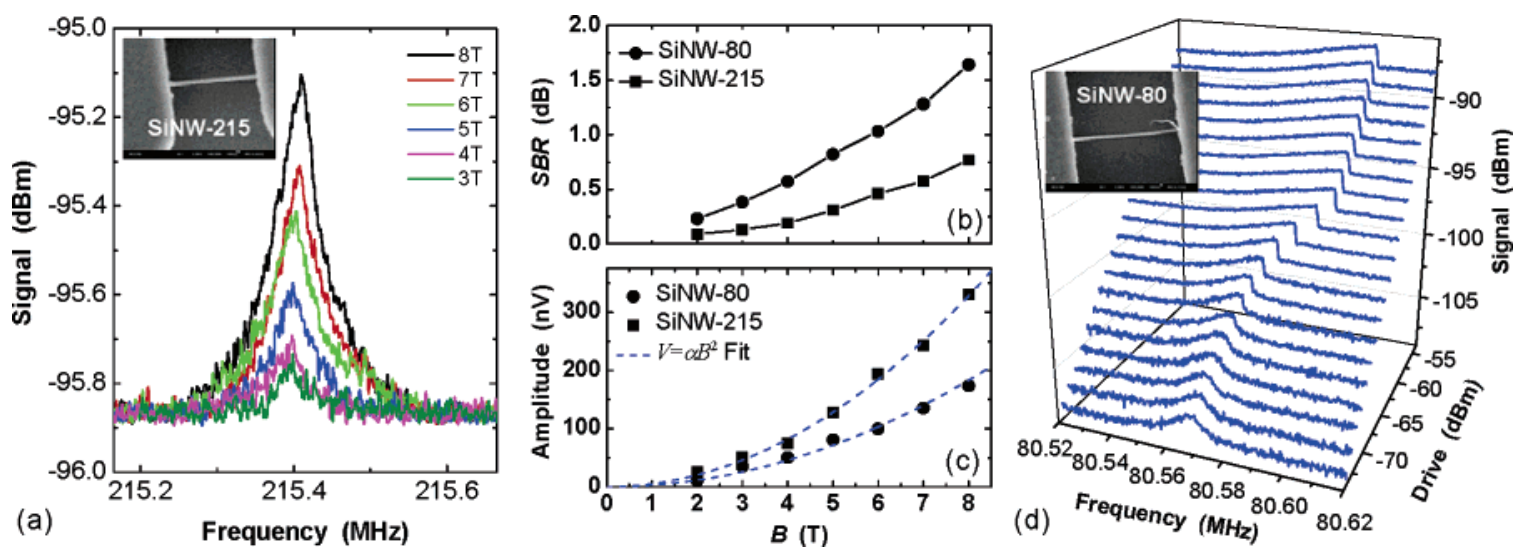

Figure 3. Measured resonance characteristics of the pristine (nonmetallized) high-resistance SiNW VHF resonators. (a) Magnetomotive response (RTI) from the SiNW-215 device at varying $B$ field (RF drive to device is $-42 \mathrm{dBm}$ ). (b) Detection efficiency (SBR) achieved in the measurement and (c) the actual voltage signal amplitude of the SiNW resonances, again proportional to $B^{2}$ as expected. (d) Response (RTI) from the SiNW-80 device as the RF drive to device is varied from -74 to $-54 \mathrm{dBm}$ (at $B=8 \mathrm{~T}$ ).

quency pulling is a stiffening effect arising from the Duffing instability in doubly clamped beams and wires. ${ }^{22}$ This canonical response, originating from tension induced at large lateral displacements (high vibration amplitudes), provides evidence that the SiNWs and their self-welded clamped ends are indeed robust.

We now discuss measurements on VHF resonators based on thinner, pristine (unmetallized) SiNWs. These SiNWs have high two-terminal resistances; we minimize the effect of the significant impedance mismatch by carefully nulling the background at the readout port. ${ }^{5}$ Figure 3 a shows data (RTI) from SiNW-215, which is a $3.2 \mathrm{k} \Omega$ pristine $\mathrm{SiNW}$ with dimensions $L=1.69 \mu \mathrm{m}, d=81 \mathrm{~nm}$, and measured $f_{0}$ $\approx 215 \mathrm{MHz}$. With $P_{\mathrm{drv}}=-42 \mathrm{dBm}$ sent to drive the device, the lowest nulled background level is $-95.87 \mathrm{dBm}(0.26$ $\mathrm{pW}$ ), and the peak power on resonance at $8 \mathrm{~T}$ is $-95.1 \mathrm{dBm}$ $(0.31 \mathrm{pW})$; these correspond to an $\sim 0.8 \mathrm{~dB}$ SBR and a NEMS resonance signal power of $50 \mathrm{fW}$. Similarly, for another device SiNW-80, a $3.6 \mathrm{k} \Omega$ pristine SiNW with $L=$ $2.77 \mu \mathrm{m}, d=74 \mathrm{~nm}$, and $f_{0} \approx 80 \mathrm{MHz}$, the background is nulled to $-108 \mathrm{dBm}(16 \mathrm{fW})$ with $P_{\mathrm{drv}}=-74 \mathrm{dBm}$. At $B$ $=8 \mathrm{~T}$, the peak signal power is $-106.4 \mathrm{dBm}(23 \mathrm{fW})$, yielding $\mathrm{SBR}=1.6 \mathrm{~dB}$ and a resonance signal power of 7 $\mathrm{fW}$. Although the actual resonance signal of SiNW-215 is larger, in readout, its SBR is lower than that of SiNW-80 because a much lower background level has been achieved around $80 \mathrm{MHz}$ than around $215 \mathrm{MHz}$. This is also shown in Figure 3b,c. By fitting the resonance amplitude of the rms voltage signal to a $B^{2}$ dependence as shown in Figure 3c, we determine $I_{\mathrm{rms}}\left(\omega_{0}\right)=14 \mathrm{nA}$ for SiNW-215 with a measured $Q \approx 5750$, and $I_{\mathrm{rms}}\left(\omega_{0}\right)=0.65 \mathrm{nA}$ for SiNW-80 with $Q \approx 13100$. Compared to their metallized counterparts, the high-resistance pristine SiNWs carry much lower current. Hence, a much weaker Lorentz force is induced and this, in turn, generates a much smaller RF signal output. Nevertheless, the achieved SBRs of $0.8 \mathrm{~dB}$ at $215 \mathrm{MHz}$ and $1.6 \mathrm{~dB}$ at $80 \mathrm{MHz}$ are significantly higher than the previously obtained $0.2 \mathrm{~dB}$ SBR at the $25.6 \mathrm{MHz}$ resonance from a $2.14 \mathrm{k} \Omega$ doped silicon beam. ${ }^{20}$ Nonlinear operation of pristine SiNW resonators have also been demonstrated, as illustrated in Figure 3d with data from the SiNW-80 resonance, with increasing RF drive from -74 to $-54 \mathrm{dBm}$ at fixed $B=8 \mathrm{~T}$.

High $Q$ 's for SiNW resonators are important both for sensing and device applications and also to enable exploration of the origin of dissipation sources. We deduce the measured $Q$ from the signal seen by the first-stage amplifier using the model underlying eqs 2 and 3 . Figure 4 a displays $Q \approx 5750$ of SiNW-215 and $Q \approx 13100$ of SiNW-80. The pristine SiNW-215 exhibits a $Q$ of $\sim 2.3-3.0$ times higher than the $Q$ 's of 2500 and 2000 from the metallized SiNW-M188 and SiNW-M200 despite their similar dimensions and resonance frequencies. This difference may arise from metallizationinduced losses such as surface and interface modification and internal friction in the metal layers.

Figure $4 \mathrm{~b}$ presents the measured $Q$ 's of the SiNWs and a comparison of their performance to other types of NEMS resonators such as bottom-up Pt nanowires $(\mathrm{PtNW})^{23}$ and top-down $\mathrm{SiC}$ beams. ${ }^{24}$ These are loaded $Q$ 's, that is, measured while embedded in the measurement circuitry in an applied magnetic field $(B=6 \mathrm{~T})$. For both metallized and pristine SiNWs, we find that $Q$ decreases with increasing resonance frequency $f_{0}$. This trend has also been observed with top-down NEMS resonators. ${ }^{15}$ The product $f_{0} \times Q$ is commonly employed as a figure of merit to evaluate resonator performance. As illustrated by the $f_{0} \times Q$ contours, our SiNWs have $f_{0} \times Q$ in the $10^{11}-10^{12} \mathrm{~Hz}$ range, with SiNW-215 having $f_{0} \times Q=1.24 \times 10^{12} \mathrm{~Hz}$. These products are comparable to those of the best top-down SiC UHF NEMS ${ }^{24}$ and state-of-the-art MEMS beam resonators. ${ }^{25}$ Note that this is comparable to $f_{0} \times Q$ 's recently reported for lower frequency, but higher $Q$, NEMS resonators under tension. ${ }^{26}$

As shown in Figure 4c, a clear correlation between the measured $Q$ and device aspect ratio (length/width) is observed; larger aspect ratios yield higher $Q$ 's. This suggests that clamping losses are important in our present geometries. Previously, we found that the data from in-plane UHF SiC NEMS beams ${ }^{23}$ fit well with the theoretical prediction $Q \sim$ $(L / w)^{3} \cdot{ }^{27}$ Here, with the thinner SiNWs, we have a much 

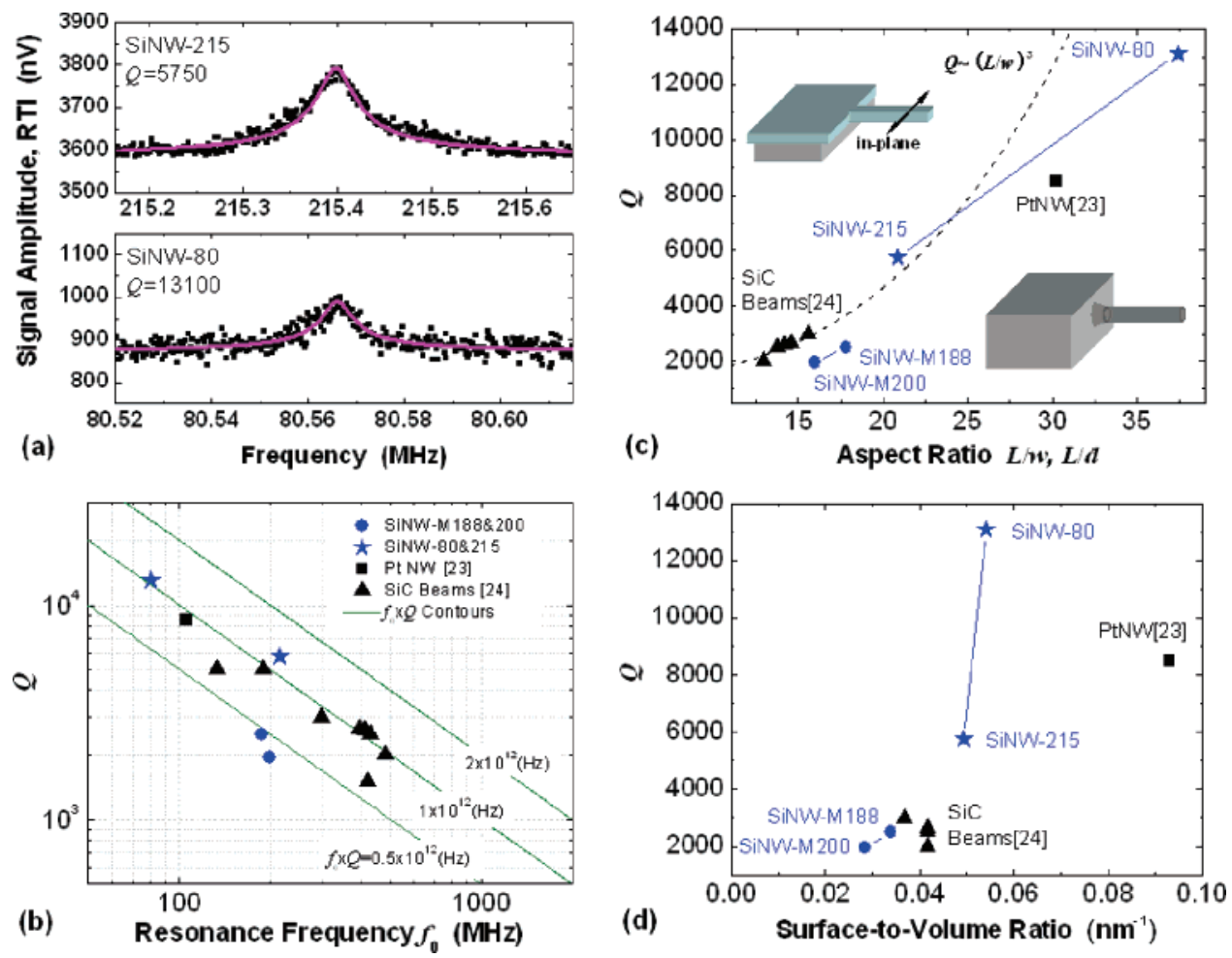

Figure 4. Quality factors ( $Q$ 's) of pristine and metallized VHF SiNW resonators. (a) Resonance signals (RTI) with high $Q$ 's from the pristine SiNWs (both data at $B=6 \mathrm{~T}$, squares are data points and solid lines are fit from the damped simple harmonic oscillator model). Measured $Q$ 's as functions of (b) device resonance frequency, (c) aspect ratio, and (d) surface-to-volume ratio, in comparison with other previously measured NEMS devices under similar experimental conditions with the same transduction scheme. Insets in (c): schematics showing the difference in clamping structures of the in-plane top-down NEMS resonators (upper left) and the pristine suspended SiNWs (bottom right).

Table 1. Characteristics and Performance Specifications of VHF SiNW Resonators ${ }^{a}$

\begin{tabular}{lllll}
\hline & SiNW-M200 & SiNW-M188 & SiNW-215 & SiNW-80 \\
\hline$L(\mu \mathrm{m})$ & 2.25 & 2.1 & 1.69 & 2.77 \\
$d(\mathrm{~nm})$ & 142 & 118 & 81 & 74 \\
metallization & & $30 \mathrm{~nm} \mathrm{Al}+5 \mathrm{~nm}$ Ti & & \\
resistance $(\Omega)$ & $\sim 50$ & 180 & $3.2 \mathrm{k}$ & $3.6 \mathrm{k}$ \\
$f_{0}(\mathrm{MHz})$ & 199.68 & 2500 & 215.40 & 80.57 \\
$Q$ & 2000 & $4.70 \times 10^{11}$ & 5750 & $1.24 \times 10^{12}$ \\
$f_{0} \times(\mathrm{Hz})$ & $3.99 \times 10^{11}$ & 45.2 & 17.1 & $1.06 \times 10^{12}$ \\
$M_{\text {eff }}(\mathrm{fg})$ & 70.1 & 62.9 & 31.4 & 23.4 \\
$k_{\text {eff }}(\mathrm{N} / \mathrm{m})$ & 110.3 & 187 & 152 & 6.0 \\
$E_{\mathrm{Y}}(\mathrm{GPa})$ & 182 & & & 184
\end{tabular}

${ }^{a}$ The SiNWs are named by their nominal resonance frequency in MHz, with "M" denoting the metallized ones.

wider aspect ratio range where it appears that likely the $Q$ 's of SiNWs do not scale as aggressively as $Q \sim(L / w)^{3}$. We suspect that the precise geometry of the NW clamping, as depicted by the schematic bottom-right inset in Figure 4c, plays an important role here. The support region for an inplane beam is a plate as thick as the beam but much wider (upper-left inset in Figure 4c). This may provide much stiffer clamping (more isolation) for in-plane motion than for outof-plane motion. These considerations lead to clampinglosses scaling as $Q \sim(L / w)^{3}$ and $Q \sim(L / w)$, respectively. ${ }^{27}$ The actual points of NW attachment, shown in SEM images of Figures 1-3 and also more carefully inspected in ref 8, have conical structure and sometimes a backward-growing parasitic wire at one end. Presumably, the resulting isolation is less than achieved for in-plane beams but may be comparable to that for out-of-plane beams. Nonetheless, single-crystal pristine SiNWs with large aspect ratio $(L / d)$ in the range of 20-40 do exhibit very high $Q$ 's. Figure $4 \mathrm{~d}$ shows that $Q$ for similar devices (either metallized or pristine) does not decrease with increasing surface-to-volume ratio. This suggests that possible surface loss phenomena, if present, are overwhelmed by large clamping losses, and hence it is the latter that accounts for the observed change of $Q$ from one device to another in the same category 


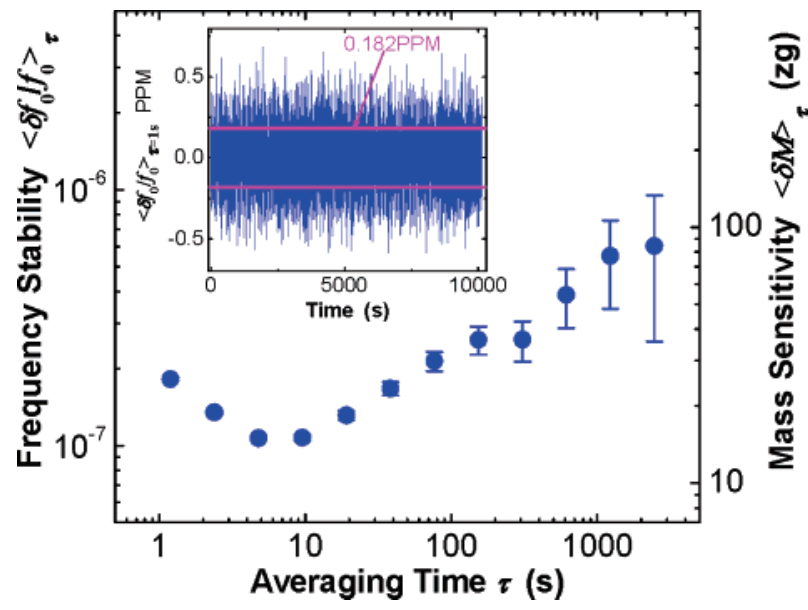

Figure 5. Measured fractional resonance frequency fluctuation and mass sensitivity of the metallized SiNW-M200 device as a function of the averaging time employed in the measurement. Inset: The "instantaneous" fractional resonance frequency fluctuation with 1 s averaging time.

(metallized or pristine). Even with larger surface-to-volume ratios, the pristine SiNWs have $Q$ 's a few times higher than obtained from metallized devices. This implies that processrelated (top-down vs bottom-up) surface modifications can indeed affect the $Q$ 's more than geometric scaling of surface losses.

Table 1 presents representative attributes and performance of several of the VHF SiNW resonators we have investigated to date. An immediate application of our dynamical measurements is the evaluation of basic mechanical properties of epitaxial SiNWs. Instead of using an atomic force microscope (AFM) to perform what are often arduous nanowire bending experiments, we are instead able to carry out resonance measurements to determine the elastic properties of these SiNWs. This is both convenient and accurate; resonance frequency can be measured with high precision and the uncertainty in deducing elastic properties comes solely from dimensional measurements. As an example, we extract the Young's modulus, $E_{\mathrm{Y}}$, from eq 1 for each SiNW resonator operating in the linear regime of motion. For metallized SiNWs, a correction factor $\left[M /\left(M+M_{\mathrm{m}}\right)\right]^{1 / 2}$, where $M_{\mathrm{m}}$ is the mass of the metallization layer and $M$ the mass of the SiNW, denormalizes the effects of the overlayer mass loading. We find $E_{\mathrm{Y}}=182 \pm 16 \mathrm{GPa}, 186 \pm 17 \mathrm{GPa}$, $152 \pm 14 \mathrm{GPa}$, and $184 \pm 16 \mathrm{GPa}$ for SiNW-M200, SiNWM188, SiNW-215, and SiNW-80, respectively. These results are in good agreement with the widely used values in the range $\sim 160-200 \mathrm{GPa}$ for single-crystal bulk Si along the $\langle 111\rangle$ direction and also agree very well with recent AFMbased measurements. ${ }^{9}$

Given their extremely small masses and very high frequency mechanical resonances, these SiNWs make exceptionally responsive resonant sensors. An additional critical attribute for such applications is the noise performance of the sensors; for frequency-shift-based detection, resonator phase noise is key. Accordingly, we have performed initial experimental investigations of the frequency stability of these SiNW resonators. We embed a SiNW resonator into a low- noise phase-locked loop circuit to implement real-time frequency tracking. ${ }^{2,28}$ This allows us to characterize the frequency stability by measuring the fractional resonance frequency fluctuation $\left\langle\delta f_{0} / f_{0}\right\rangle_{\tau}$ as a function of the averaging time $\tau$ employed for the measurement. When used for mass sensing, the device's actual mass sensitivity is also, therefore, a function of the measurement time, $\langle\delta M\rangle_{\tau}=2 M_{\mathrm{eff}}\left\langle\delta f_{0} / f_{0}\right\rangle_{\tau}$. Figure 5 shows the measured $\left\langle\delta f_{0} \mid f_{0}\right\rangle_{\tau}$ and $\langle\delta M\rangle_{\tau}$ from SiNWM200. For $\tau=1 \mathrm{~s}$, the measured rms frequency fluctuation is $\left\langle\delta f_{0} / f_{0}\right\rangle_{\tau=1 s}=0.182 \mathrm{ppm}$. Given the device's exceptional mass responsivity ${ }^{28} \mathscr{R}=f_{0} /\left(2 M_{\text {eff }}\right)=1.4 \mathrm{~Hz} / \mathrm{zg}$ and its demonstrated excellent frequency stability, this noise level equates to a mass sensitivity of $\langle\delta M\rangle_{\tau=1 s}=25.5 \mathrm{zg}(1 \mathrm{zg}=$ $10^{-21} \mathrm{~g}$ ). This is comparable to the mass sensitivity of stateof-the-art, top-down VHF resonators, such as the $190 \mathrm{MHz}$ $\mathrm{SiC}$ device recently employed to realize the experimental milestone of zeptogram-scale real-time mass sensing. ${ }^{2}$ Characterization and analysis of noise and stability for these SiNWs are more extensively detailed elsewhere. ${ }^{29}$

In conclusion, we have demonstrated that epitaxially grown single-crystal SiNWs can be engineered to realize robust NEMS resonators operating at frequencies in the VHF range and higher. These bottom-up SiNW resonators exhibit excellent performance; here we demonstrate high $Q$ 's at VHF and zeptogram mass sensitivity. Pristine, unmetallized SiNWs provide very high $Q$ 's and $f_{0} \times Q$ products, however, their high performance comes at the cost of a significant impedance mismatch to $50 \Omega$. Metallized SiNWs, on the other hand, provide an excellent low-impedance RF match but have lower $Q$ 's arising, presumably, from additional damping induced by their metallic overlayers. We anticipate that it is feasible to achieve both high- $Q$ and optimal (50 $\Omega$ ) impedance matching by metamorphosing single-crystal, semiconducting SiNWs into metallic structures, e.g., NiSi NWs. ${ }^{30}$

A principal advantage of the suspended SiNW resonators developed in this work is their ease of fabrication and high yield. By pushing the dimensions of the microtrenches downward and simultaneously optimizing the NW growth conditions, we expect that smaller, even molecular-size, suspended SiNWs should be achievable. These will enable scaling fundamental resonance frequencies into the extreme $\mathrm{UHF}$ and low microwave range. Given their $\mathrm{pW}$ - to sub$\mathrm{pW}$ operating power levels, SiNW resonators will enable future ultralow power applications such as high frequency mechanical signal processing, ${ }^{25}$ mechanical logic applications, ${ }^{6}$ and electromechanical qubits. ${ }^{4}$ The suspended NEMS nanowire technology described herein, combined with the recent development of controlled epitaxial growth of selfaligned SiNW arrays, ${ }^{31}$ should also now make it feasible to engineer SiNW-based resonator arrays to enable very-largescale nanomechanical device integration.

Acknowledgment. We gratefully acknowledge support from the NSF under grant EECS 0425914 (NSF-NSEC), MARCO, and from DARPA/SPAWAR under grant N6600102-1-8914. X.L. Feng thanks S. Stryker for help in engineering and fabrication of experimental apparatus, and B. Gudlewski for helpful discussions. 


\section{References}

(1) Ekinci, K. L.; Roukes, M. L. Rev. Sci. Instrum. 2005, 76, 061101.

(2) Yang, Y. T.; Callegari, C.; Feng, X. L.; Ekinci, K. L.; Roukes, M. L. Nano Lett. 2006, 6, 583.

(3) Rugar, D.; Budakian, R.; Mamin, H. J.; Chui, B. W. Nature 2004 $430,329$.

(4) Schwab, K. C.; Roukes, M. L. Phys. Today 2005, July, 36.

(5) Feng, X. L.; White, C. J.; Hajimiri, A.; Roukes, M. L. 2007, submitted for publication.

(6) Roukes, M. L. In Technical Digest 2004 IEEE International Electron Devices Meeting, San Francisco, CA, December 13-15, 2004; p 539

(7) Cleland, A. N.; Roukes, M. L. Appl. Phys. Lett. 1996, 69, 2653.

(8) He, R. R.; Gao, D.; Fan, R.; Hochbaum, A. I.; Carraro, C.; Maboudian, R.; Yang, P. D. Adv. Mater. 2005, 17, 2098.

(9) San Paulo, A.; Bokor, J.; Howe, R. T.; He, R.; Yang, P.; Gao, D.; Carraro, C.; Maboudian, R. Appl. Phys. Lett. 2005, 87, 053111.

(10) He, R. R.; Yang, P. D. Nat. Nanotechnol. 2006, 1, 42.

(11) Karabacak, D.; Kouh, T.; Huang, C. C.; Ekinci, K. L. Appl. Phys. Lett. 2006, 88, 193122

(12) Truitt, P. A.; Hertzberg, J. B.; Huang, C. C.; Ekinci, K. L.; Schwab, K. C. Nano Lett. 2007, 7, 120.

(13) Knobel, R.; Cleland, A. N. Appl. Phys. Lett. 2002, 81, 2258.

(14) Cleland, A. N.; Roukes, M. L. Sens. Actuators 1999, 72, 256.

(15) Huang, X. M. H.; Feng, X. L.; Zorman, C. A.; Mehregany, M.; Roukes, M. L. New J. Phys. 2005, 7, 247.

(16) Knobel, R.; Cleland, A. N. Nature 2003, 424, 291.

(17) LaHaye, M. D.; Buu, O.; Camarota, B.; Schwab, K. C. Science 2004, 304, 74.

(18) Cleland, A. N.; Aldridge, J. S.; Driscoll, D. C.; Gossard, A. C. Appl. Phys. Lett. 2002, 81, 1699.
(19) Flowers-Jacobs, N. E.; Schmidt, D. R.; Lehnert, K. W. Phys. Rev. Lett. 2007, 98, 096804.

(20) Ekinci, K. L.; Yang, Y. T.; Huang, X. M. H.; Roukes, M. L. Appl. Phys. Lett. 2002, 81, 2253.

(21) Huang, X. M. H.; Zorman, C. A.; Mehregany, M.; Roukes, M. L. Nature 2003, 421, 496.

(22) Postma, H. W. C.; Kozinsky, I.; Husain, A.; Roukes, M. L. Appl. Phys. Lett. 2005, 86, 223105.

(23) Husain, A.; Hone, J.; Postma, H. W. C.; Huang, X. M. H.; Drake, T.; Barbic, M.; Scherer, A.; Roukes, M. L. Appl. Phys. Lett. 2003, $83,1240$.

(24) Feng, X. L.; Zorman, C. A.; Mehregany, M.; Roukes, M. L. In Technical Digest 2006 Solid-State Sensors, Actuators \& Microsystems Workshop, Hilton Head, SC, June 4-8, 2006; p 86.

(25) Nguyen, C. T. C. In Proceedings of the 2005 IEEE International Frequency Control Symposium, Vancouver, Canada, August 2931, 2005; p 1.

(26) Verbridge, S. S.; Shapiro, D. F.; Craighead, H. G.; Parpia, J. M. Nano Lett. 2007, 7, 1728.

(27) Lifshitz, R.; Cross, M. C. Phys. Rev. B 2001, 64, 085324.

(28) Ekinci, K. L.; Yang, Y. T.; Roukes, M. L. J. Appl. Phys. 2004, 95, 2682.

(29) Feng, X. L; He, R. R.; Yang, P. D.; Roukes, M. L. In Digest of Technical Papers, The 14th International Conference on Solid-State Sensors, Actuators and Microsystems (Transducers '07), Lyon, France, June 10-14, 2007; p 327.

(30) Wu, Y.; Xiang, J.; Yang, C.; Lu, W.; Lieber, C. M. Nature 2004, $430,61$.

(31) Hochbaum, A. I.; Fan, R.; He, R. R.; Yang, P. D. Nano Lett. 2005 $5,457$.

NL0706695 